This item was submitted to Loughborough's Research Repository by the author.

Items in Figshare are protected by copyright, with all rights reserved, unless otherwise indicated.

\title{
Optical diagnostics studies of air flow and powder fluidisation in Nexthaler®. Part II: Use of fluorescent imaging to characterise transient release of fines from a dry powder inhaler
}

\section{PLEASE CITE THE PUBLISHED VERSION}

https://doi.org/10.1016/j.jpharm.2018.07.032

\section{PUBLISHER}

(C) Elsevier

\section{VERSION}

AM (Accepted Manuscript)

\section{PUBLISHER STATEMENT}

This work is made available according to the conditions of the Creative Commons Attribution-NonCommercialNoDerivatives 4.0 International (CC BY-NC-ND 4.0) licence. Full details of this licence are available at: https://creativecommons.org/licenses/by-nc-nd/4.0/

\section{LICENCE}

CC BY-NC-ND 4.0

\section{REPOSITORY RECORD}

Merusi, C., G. Brambilla, Edward J. Long, Graham K. Hargrave, and Hendrik K. Versteeg. 2019. "Optical Diagnostics Studies of Air Flow and Powder Fluidisation in Nexthaler ${ }^{\circledR}$. Part II: Use of Fluorescent Imaging to Characterise Transient Release of Fines from a Dry Powder Inhaler". figshare.

https://hdl.handle.net/2134/34815. 
Optical diagnostics studies of air flow and powder fluidisation in Nexthaler ${ }^{\circledR}$

Part II: Use of fluorescent imaging to characterise transient release of fines from a dry powder inhaler

Merusi C. ${ }^{1}$, Brambilla G. ${ }^{1}$, Long E.J. ${ }^{2}$, Hargrave G.K. ${ }^{2}$, Versteeg H.K. ${ }^{2 \dagger}$

${ }^{1}$ Chiesi Farmaceutici S.p.A., 43122 Parma, Italy.

${ }^{2}$ Wolfson School of Mechanical, Electrical and Manufacturing Engineering, Loughborough University, Loughborough, LE11 3TU, U.K.

† Corresponding author: H.K.Versteeg, e-mail: H.K.Versteeg@Lboro.ac.uk 


\begin{abstract}
The fine particle fraction is a key indicator of therapeutic effectiveness of inhaled pharmaceutical aerosols. This paper presents a fluorescence imaging technique to visualise and characterise the emission of active pharmaceutical ingredient (API) fines in model formulations containing coarse lactose carrier and 1.5-2 $\mu \mathrm{m}$ diameter fluorescent microspheres (model API fines). A two-camera arrangement was used to acquire simultaneous images of spatial and temporal distribution of model API fines and fluidised powder formulation near the mouthpiece exit of a DPI. Digital image analysis showed that the model API fines were released along with the bulk of the powder dose. More rapidly accelerating airflows were found to cause earlier release of API fines. The fluorescence imaging technique analyses a substantial fraction of the aerosol plume and was found to provide effective time-resolved characterisation of the de-aggregation and release of API fines with consistent results across a wide range of model API concentrations. Future studies should demonstrate the usefulness of the fluorescence imaging technique across different formulations and DPI devices.
\end{abstract}

Keywords: powder fluidization; dry powder inhaler; optical diagnostics; high-speed fluorescence imaging; model API; de-aggregation 


\section{Introduction}

Dry powder inhalers (DPI) deliver small quantities of drug in powder form for the treatment of respiratory diseases, such as asthma and COPD, and for systemic delivery of proteins and peptides. For effective drug delivery to the lungs, particles of the active pharmaceutical ingredient (API) in the air stream must be sufficiently small $(1-5 \mu \mathrm{m})$ to penetrate the lower airways. However, such small particles are highly cohesive and must be formulated to produce particles with a size at least $40-50$ $\mu \mathrm{m}$ so that the resulting powder has good flowability and can be aerosolised effectively. This may be achieved by (i) processing to generate self-agglomerates of essentially pure drug, (ii) processing with inert excipient particles of a similar size range to generate composite agglomerates, or, (iii) blending with large non-active carrier particles, e.g. lactose, to which the drug particles adhere, (Newman \& Busse, 2002; Chan \& Chew, 2003; Laube et al, 2011). Powder emission from DPIs is a breath-actuated fluidisation and particle transport process, which is initiated by the patient sucking on the device mouthpiece. A sufficiently powerful inhaled air stream is needed to fluidise the powder and to generate the disruptive forces to overcome the inter-particulate adhesive/cohesive forces within the agglomerates to release inhalable-size active drug particles before the aerosol enters the patient's airways (Finlay, 2001; Voss \& Finlay, 2002; Coates et al, 2005; Nichols \& Wynn, 2008; Zhou et al, 2014).

The powder fluidisation and de-aggregation processes leading to the release of API fines in DPIs are at present poorly understood. Optical diagnostics are powerful techniques with considerable potential for the study of turbulent air flows and powder fluidisation with relevance to pharmaceutical inhalers. Tuley et al (2008) used high-speed imaging in conjunction with white light illumination in a fundamental study of fluidisation of powders with different bulk properties in a transparent, idealised DPI. The work revealed two distinctly different fluidisation regimes: (i) 'erosion' and (ii) 'fracture', which depended on the powder type. Image analysis enabled quantification of the fluidised fraction as a function of time for different air pressures. It was shown that lactose powders were fluidised by means of the 'fracture' regime and that the fluidised fraction depended only on instantaneous air pressure for these powders. Particle image velocimetry (PIV) has recently been used (Ngoc et al, 2013) to carry out a systematic evaluation of the effect of geometry on the flow environment within the de-agglomeration chamber of an idealised DPI. Detailed study of the distribution of turbulence intensity yielded optimal de-agglomeration grid structure and dimensions as well as optimised de- 
agglomeration chamber length and volume of the idealised DPI. Increases of the turbulence intensity were broadly found to correlate with improved aerosolisation and de-agglomeration of a budesonide-lactose blend. Pasquali et al (2015) used high-speed imaging and PIV to characterise the air flow inside a Nexthaler ${ }^{\circledR}$ DPI and outside its mouthpiece. They also observed powder fluidisation and aerosol transport processes inside the device. The study examined the effects of acceleration to steady state inhalation rates $\left(40-801 . \mathrm{min}^{-1}\right.$, rise time $\left.0.3-1.2 \mathrm{~s}\right)$ on the temporal discharge of a proprietary lactose inhalation powder placebo, comprising coarse carrier particles blended with fine excipient particles. Imaging studies within the device allowed observation of the fluidisation process in situ. PIV studies showed that high swirl levels are generated in the swirl chamber of the device. This was observed to produce a highly turbulent swirling flow at the device mouthpiece, which interacted with the stationary air just outside the mouthpiece, generating additional turbulence. The aerosol emission process at the mouthpiece exit was observed to comprise two phases: (i) the main powder emission event consisting of a mixture of coarse and fine particles, (ii) a second emission peak containing mainly coarse carrier particles.

One drawback of the optical set-up used in PIV studies is that the scattered light cannot discriminate between (a) inhalable active drug particles, (b) excipient fines which were deliberately added to the formulation, or, (c) fines inherent within any lactose sample. The main techniques for characterising aerosols emitted from inhaler devices are Cascade Impaction (Cl), Laser Diffraction (LD) and Single Particle Aerosol Mass Spectrometry, (SPAMS):

- Cascade impactor measurements involve quantitative analysis of drug deposition on a series of impactor plates, which classify an aerosol cloud sample into discrete size fractions. This allows determination of emitted dose, fine particle dose and particle size distribution. $\mathrm{Cl}$ procedures are detailed in the pharmacopoeias (European Directorate for Quality in Medicines and Healthcare, 2013; US Pharmacopoeia, 2013) and are the gold standard in industrial quality control as well as basic research in respiratory drug delivery. However, the technique consumes significant and costly analytical resource.

- Laser diffraction is widely used to measure size distributions of DPI aerosol plumes (e.g. Sim et al, 2014). This technique does not directly discriminate between API fines and excipient and/or carrier fines. However, Zeng et al, (2006) have established a linear correlation between the total aerosol fine fraction determined by a Malvern 2600 LD particle sizer and 
the FPF of the drug, $(\leq 6.8 \mu \mathrm{m})$, determined by impaction with a multi-stage liquid impinger (MSLI) for micronised salbutamol sulphate blends with coarse sugar carriers with added fine lactose. Thus, by reference to the impactor performance of a limited number of "calibration formulations", LD can be used for rapid estimation of fine drug fraction of multiple formulations and, hence, formulation optimisation for potential clinical study within a development programme.

- SPAMS is a laser-based mass spectrometry technology with potential capability to measure size and chemical composition of single aerosol particles. Output data is processed to provide particle size distributions for individual API components within an aerosol cloud. Applications to both DPI, (Fergenson et al, 2013; Susz et al, 2014) and pMDI systems, (Morrical et al, 2015), have been described. Recently, Jetzer et al (2017) used SPAMS to determine the size distribution and chemical composition of particles emitted by two DPI combination products, namely NEXThaler ${ }^{\circledR}$ and Diskus ${ }^{\circledR}$. The former produced significantly higher fine particle fraction $(<5 \mu \mathrm{m})$ and extrafine particle fraction $(1-2 \mu \mathrm{m})$ suggesting more effective detachment of the APIs from the lactose carrier. It must be noted that the aerodynamic particle sizing technique used by the instrument works with a relatively small sample of the aerosol cloud. Moreover, the required incorporation of a settling chamber into the apparatus is known to lead to under-sampling of larger particles due to gravitational settling. This could distort the high end of the particle size distribution and, hence, creates errors in the mass-weighted median aerodynamic diameter. Further refinement of the methodology is on-going and it is claimed that chemical identification and particle size distribution analysis for a given component can be completed within $\sim$ one hour.

All three techniques have a further limitation: the aerosol is characterised ex-mouthpiece, where information about the complex physical processes that ultimately control the aerodynamic performance of the aerosol (powder fluidisation, de-agglomeration and aerosol transport) has been lost. To address this issue, the present work proposes the use of fluorescence imaging to study deaggregation and release of fines from a DPI. Fluorescent tracer dyes have been used widely in flow visualisation to enhance contrast and visibility (Merzkirch, 1987). Fluorescent seeding or tracer particles can be added to a flow, for example, to study mixing. Molecules of the seeding or tracer are excited from a ground state to a higher energy state by irradiation with laser light of a wavelength 
that matches with an absorption line of the molecule. Several competing pathways exist for the molecules to return to the ground state. The main path involves (i) relaxation to an intermediate energy state through radiative emission - fluorescent emission - and (ii) return back to the ground state by loss of further energy in the form of heat. Because the energy difference between the excited and intermediate states is smaller, the wavelength of the fluorescent light is longer than the laser irradiation. Fluorescent emission from a particle-laden flow can, therefore, be separated from scattered incident light by means of optical filters. Fluorescent particles have been widely used to study mixing processes (e.g. Towers et al. 1999). The characteristics of fluorescence-based imaging that are of interest here are:

(i) The technique is highly specific to a chemical or material,

(ii) The results are time-resolved, so aerosol generation and development can be studied in considerable,

(iii) The technique is a planar whole-field method, and hence, a substantial fraction of a pharmaceutical aerosol cloud is observed at every instant in time.

The work reported in this paper is the second part of a two-part study of powder fluidisation in prototype Nexthaler® DPI. The first (Pasquali et al, 2015) focused on powder dose emission from the device as a function of inspiratory effort inhaled air flow rate. The present work considered the emission of API fines: the temporal characteristics of de-aggregation and release of a proprietary placebo powder. Fluorescent microspheres with diameters in the respirable range 1-5 $\mu \mathrm{m}$ were used as a surrogate for the API fines and blended with the Nexthaler ${ }^{\circledR}$ placebo powder. The optical diagnostics rig described in Pasquali et al (2015) was modified to allow simultaneous imaging of total scattered light (coarse carrier, excipient and API surrogate) and fluorescent light, (API surrogate only). Differential detection of the fluorescent microspheres within the aerosolised cloud enables in situ study of powder fluidisation and release of model API fines. 


\section{Materials and Methods}

\subsection{Materials}

\subsubsection{Fluorescent Microspheres}

Commercially available red fluorescent polymer microspheres (Cospheric LLC, Santa Barbara, CA, USA) were used as the API surrogate in the present work. They are fabricated from a proprietary polymer with density $1.3 \mathrm{~g} \cdot \mathrm{cm}^{-3}$ and show peak fluorescence emission at $607 \mathrm{~nm}$ from excitation at $527 \mathrm{~nm}$ (Cospheric Inc., 2018). According to manufacturer's data, the microspheres have the following particle size distribution: $d_{50}=1.5-2.0 \mu \mathrm{m}, d_{95}=3.5 \mu \mathrm{m}$ and $d_{99}=5 \mu \mathrm{m}$, so $99 \%$ of the distribution is within the target range of inhalable particles.

\subsubsection{Fluorescent Microsphere Powder Formulations}

Chiesi's Nexthaler ${ }^{\circledR}$ inhalation powder formulations comprise active pharmaceutical ingredients, (APIs), blended with a proprietary lactose-based carrier vehicle. This carrier system is robust and can be manufactured at laboratory scale $(\geq 50 \mathrm{~g})$ or pilot/industrial scale, $(\geq 1.0 \mathrm{~kg})$, without perturbing the aerosolisation performance of an active-vehicle blend. Batches of the powder formulation were blended with fluorescent microspheres to nominal concentrations of $0.02 \% \mathrm{w} / \mathrm{w}$, $0.05 \% \mathrm{w} / \mathrm{w}, 0.25 \% \mathrm{w} / \mathrm{w}, 1.00 \% \mathrm{w} / \mathrm{w}$ and $2.00 \% \mathrm{w} / \mathrm{w}$, respectively. All formulation preparation involved "layering" the microsphere sample between aliquots of vehicle within a customised vessel held within the chamber of a Turbula powder blender, (model T2F, Glen Creston, Middlesex, UK), and blending at $34 \mathrm{rpm}$ for 90 minutes. The presence of fluorescent microspheres on the lactose carrier surface was confirmed by confocal fluorescent microscopy, (Fig. 1).

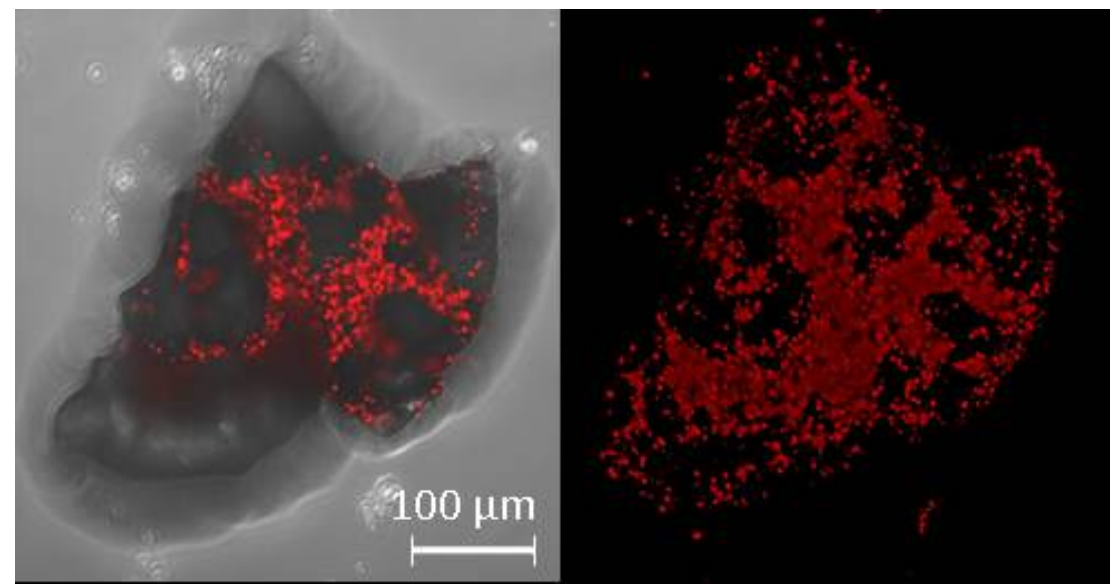

Figure 1: Confocal microscopy image of lactose carrier surface with $1 \% \mathrm{w} / \mathrm{w}$ fluorescent microspheres 


\subsection{Methods}

\subsubsection{Prototype Nexthalers}

Pre-production prototypes of the Nexthaler ${ }^{\circledR}$ DPI, (Corradi et al, 2014), were assembled from unfilled pieces as described by Pasquali et al (2015), and hand filled with $\sim 1.5 \mathrm{~g}$ of the required powder formulation. A different DPI prototype unit was filled for each powder with a particular concentration of model API fines. The aerodynamic performance of the aerosolised fluorescent microsphere powders (MMAD and FPF) was compared with the standard formulation containing BDP active using the Next Generation Impactor (NGI) at a rate of $601 /$ min with a 4 s suction duration. A fluorimeter (Jenway series 6285) was used to determine the relative mass of fluorescent microspheres on each impaction plate, with a 380-500nm bandpass filter used for excitation and a $545 \mathrm{~nm}$ high-pass cut-off filter used for emission. The gain was set to $40 \%$ for all calibration and test samples. Three repeat tests were carried out on the fluorescent microsphere formulations. Selected results from Pasquali et al (2012) are shown in Table 1.

\begin{tabular}{|l|c|c|}
\hline & $\begin{array}{c}\text { Active formulation } \\
\text { BDP 1\% w/w }\end{array}$ & $\begin{array}{c}\text { Fluorescent microsphere } \\
\text { formulation 1\% w/w }\end{array}$ \\
\hline Fine particle fraction $\left(\mathrm{FFP}, \mathrm{d}_{\mathrm{p}} \leq 5 \mu \mathrm{m}\right)$ & $58.5 \pm 0.5$ & $58.1 \pm 0.5$ \\
\hline $\begin{array}{l}\text { Mass median aerodynamic diameter } \\
(\mathrm{MMAD} \text { in } \mu \mathrm{m})\end{array}$ & $1.5 \pm 0.1$ & $1.6 \pm 0.1$ \\
\hline
\end{tabular}

Table 1: Comparison of FFP and MMAD of lactose/BDP formulation and formulation with fluorescent microsphere model API fines (test conditions: $\mathrm{NGI}$ at $60 \mathrm{l} / \mathrm{min}$ airflow, duration $4 \mathrm{~s}, \mathrm{~N}=3$ ).

\subsubsection{Optical Diagnostics Test Rig}

The experimental set-up is shown schematically in Figure 2. The pneumatic suction system is designed to produce systematic variations of the steady state air flow rate $\mathrm{Q}_{\max }$ and the rise time $T_{\text {rise. }}$ Thermal mass flowmeter, $(H)$, measures $Q_{\max }$, the value of which is set by adjustment to the variable area sonic restrictor, (D). $T_{\text {rise }}$ can be changed by altering the capacity of the variable volume unit $(\mathrm{G})$. Values of $\mathrm{Q}_{\max }$ between 40 and $120 \mathrm{I} \cdot \mathrm{min}^{-1}$ in conjunction with $\mathrm{T}_{\text {rise }}$ values between 0.3 and $1.2 \mathrm{~s}$ could be achieved with the present system. Instantaneous pressures were measured in the mouthpiece region and on the downstream side of the sonic restrictor with pressure transducers $\left(P_{u}\right)$ and $\left(P_{D}\right)$, respectively. The resulting temporal profiles of pressure $P_{u}$ showed an 
exponential decrease after the start of the air flow. The transient behaviour of the pressure and flow rate through the device will be closely correlated. As pressure $\mathrm{P}_{\mathrm{u}}$ decreases, a corresponding increase of the flow rate through the DPI device takes place. This was validated by comparison of the resulting pressure traces with integrated velocity distributions measured with PIV at the mouthpiece exit. Further details of this process and the pneumatic system can be found in Pasquali et al (2015).

The high speed imaging system was modified to capture the fluorescence and non-fluorescent scattered light signals simultaneously. The illumination source was a Nd:YLF laser with a single wavelength of $527 \mathrm{~nm}$, which is in the green part of the visible spectrum. Suitable optics were used to produce a pulsed laser light sheet, $(\mathrm{L})$, with a thickness of $1 \mathrm{~mm}$ in the vicinity of the device mouthpiece via an optical mouthpiece adapter $\left(\mathrm{J}_{\vee}, \mathrm{J}_{H}\right)$. The incident light sheet has a frequency of $2000 \mathrm{~s}^{-1}$ and 'on time' around $180 \mathrm{~ns}$. It illuminates particles entrained in the air flow and also excites the fluorescent microspheres, which emit light in the red part of the spectrum ( 607 nm).

A cube beam splitter, $(T)$, was used to enable simultaneous imaging of the same region with two Photron SA3 high-speed video cameras, $\left(N_{1}, N_{2}\right)$, both synchronised to the pulsed laser light. Optical filters between the beam-splitter and the cameras were used to separate the red fluorescence signal produced by the microspheres from the green laser light scattered by all particles, (carrier, excipient fines and microspheres). The scattered green light is directed through the short-pass filter ( $R$, cut-off $\geq 550 \mathrm{~nm}$ ), before being recorded by camera $N_{1}$. The red light emitted by the fluorescent particles is recorded by camera $N_{2}$ via the long-pass filter (Q, cut-on $\geq 575 \mathrm{~nm}$ ). The scattered green light has a much higher intensity than the fluorescent light, so a separate neutral density filter, $(S)$, is placed in front of camera, $N_{1}$, to produce an approximate match of the light intensities received by both cameras, so that the same aperture settings could be used. Two laser sheet alignments were used for the high-speed visualisation of the particles emerging from a Nexthaler® device;

(i) Axial plane: along the axis of the mouthpiece to visualise the aerosol plume along its centre line (Figure 2, Panels a \& b),

(ii) Swirl plane: perpendicular to the axis of the mouthpiece to visualise the aerosol plume in the swirl plane of the device (Figure 2, Panels c \& d). 


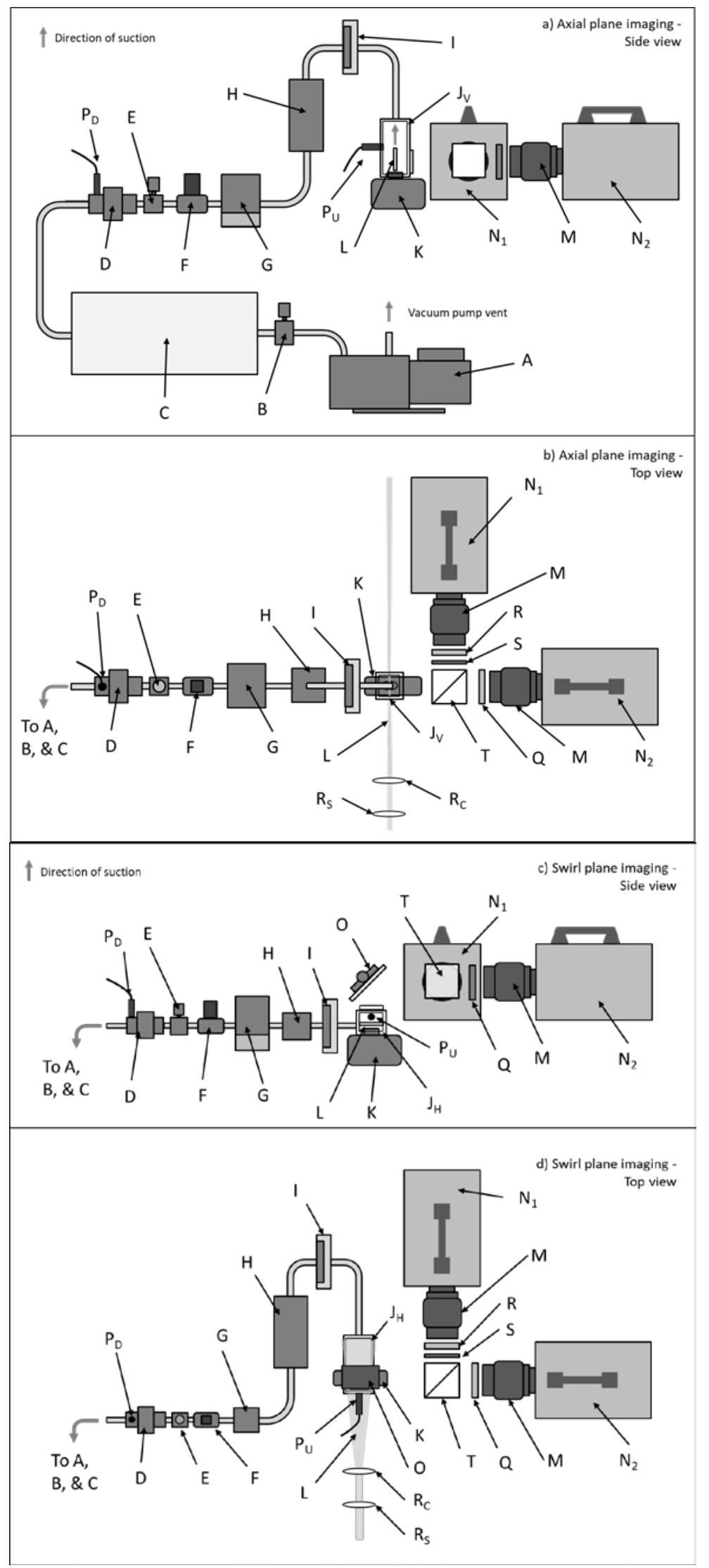

Figure 2 - Schematic of optical diagnostics test rig configurations for imaging in axial, (panels a \& b) and swirl, (panels c \& d) planes 
Key to Figure 2:
A. Vacuum pump - Edwards Speedivac ED660
B. Control valve - Legris Stainless steel ball valve
C. 20 litre steel vacuum vessel
D. Sonic Restrictor - Made in-house
E. Flow Control Valve - Legris Stainless steel ball valve
F. Rapid Switch Solenoid Controlled Ball Valve - Omal SR15 driven with 6 bar pressure
G. Variable Volume Unit - made in-house
H. Thermal Mass Flow Meter - Sierra 0-200 SI. min-1 Accuracy: 1.0\% of full scale
I. Particle Filter Housing with $1 \mu \mathrm{m}$ Particle Retention Filter - Pall Corporation type A/E

$\mathbf{J}_{\mathrm{V}}, \mathbf{J}_{\mathbf{H}}$ Custom-built adaptors with optical access - PMMA construction with glass window, internal dimensions of $28 \times 28 \times 60 \mathrm{~mm}$, two variations fitted with a silicone rubber seal where it meets the Nexthaler® Mouthpiece

K. Nexthaler® Device

L. Vertical Laser Sheet

M. Nikkor $105 \mathrm{~mm}$ macro lens

$\mathbf{N}_{1}, \mathbf{N}_{2}$. Photron APX RS High-speed cameras

O. Front coated mirror $60 \mathrm{~mm} \times 40 \mathrm{~mm}$

$\mathbf{P}_{\mathbf{D}}, \quad \mathbf{P}_{\mathbf{u}} \quad$ Pressure Transducers - Kistler 4045A5 (25 mV/bar/mA sensitivity, natural frequency $\approx 80 \mathrm{kHz}$ ) used in conjunction with a National Instruments 6110 data logger

Q. Long-pass optical filter with a cut-on at $575 \mathrm{~nm}$

R. Short-pass optical filter with cut-off at $550 \mathrm{~nm}$

S. Neutral density filter

T. Cube beam splitter 
The temporal discharge of the DPI aerosol was recorded across nine airflow suction profiles induced by accelerating the suction flow rates to pre-selected steady state values of $40,60,80 \mathrm{I} \cdot \mathrm{min}^{-1}\left(\mathrm{Q}_{\max }\right)$, in conjunction with three different rise times, $\left(T_{\text {rise }}\right), 0.3,0.7,1.2 \mathrm{~s}$, between the start of suction and the flow rate reaching its steady state value. Rationale for the choice of these airflow profiles and the resulting differential pressure profiles recorded across the device can be found in Pasquali et al (2015).

\subsubsection{Analysis of Recorded Images}

High-speed imaging of the fluidisation of dry powder formulations yielded series of image frames. Figure 3 shows a sample frame illustrating a typical spatial distribution of powder aerosol in the mouthpiece region of the DPI. To reveal details of the plume structure, the intensity fields are shown on a colour scale ranging from dark blue $=$ lowest intensity to red $=$ highest intensity. Bright regions (coloured red) indicate high local concentrations of powder particles in this image of scattered light. The temporal release of the dry powder formulation and the model API exiting the device mouthpiece was characterised by means of frame-by-frame analysis using standard digital image analysis functions available in the numerical computing environment Matlab ${ }^{\circledR}$ (The MathWorks, Inc., Natick, MA, USA). A $16 \mathrm{~mm} \times 4 \mathrm{~mm}$ analysis region close to the device mouthpiece is highlighted in Figure 3. Pixel intensities I were summed in this region, yielding $\left(\Sigma \mathrm{I}_{\mathrm{n}}\right.$ for the $\mathrm{n}^{\text {th }}$ frame of an image series. The maximum value of the sum of the pixel intensities $\left(\operatorname{MaxSum}=\operatorname{Max}\left((\Sigma l)_{n}, \mathrm{n}=\right.\right.$ $1,2, \ldots, N)$ ) was found for the entire series of $\mathrm{N}$ images representing a particular fluidisation event. Subsequently, the normalised sum of pixel intensities, defined as the ratio $(\Sigma I)_{n} /$ MaxSum, was computed for all scattered light and fluorescent images, which yielded temporal profiles of the emissions of powder formulation and of model API from the device as a function of time. 


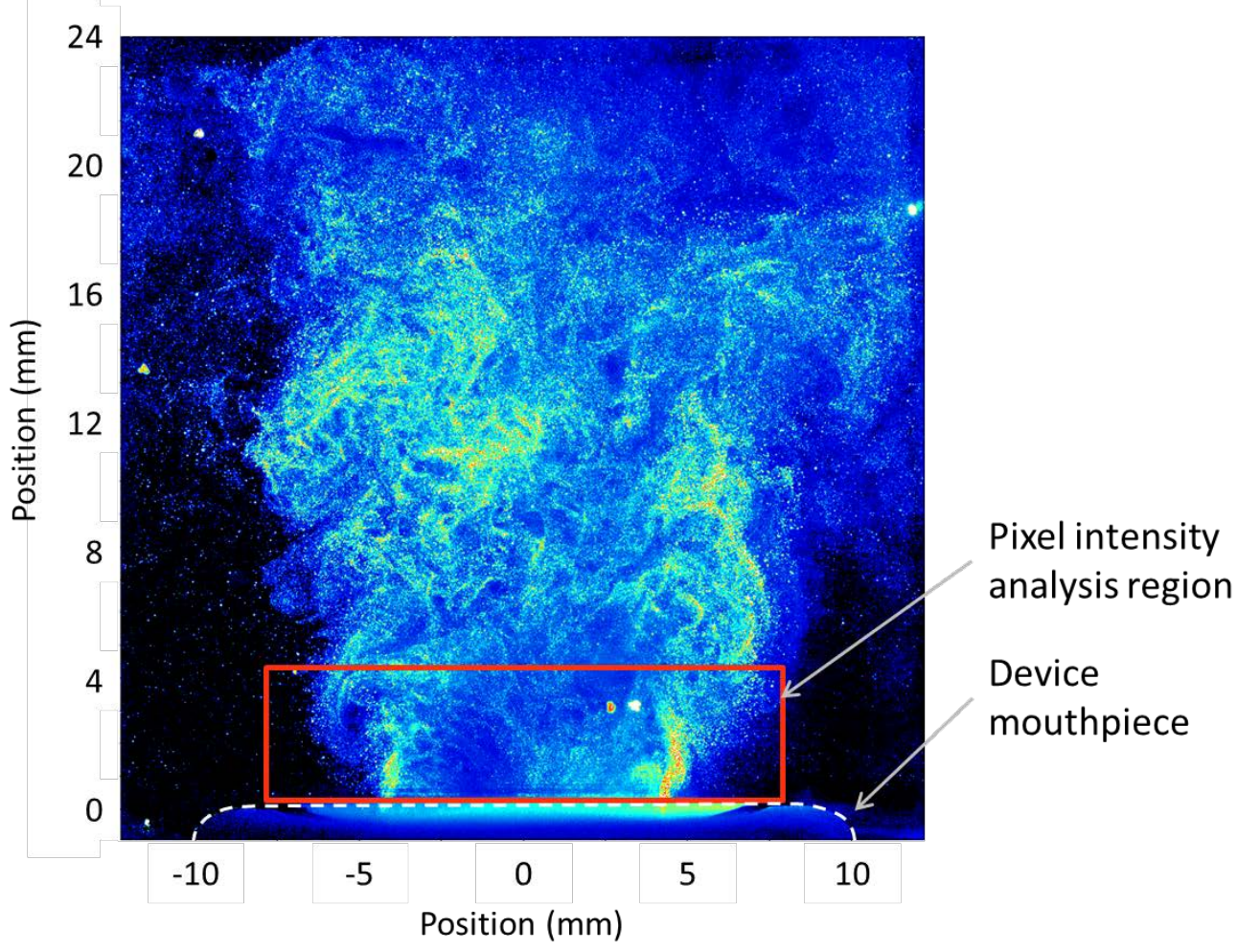

Figure 3. Sample scattered light image of aerosol plume highlighting pixel analysis region for intensity measurement 


\section{Results and Discussion}

\subsection{Fluorescent images - distribution of model API fines}

A typical set of fluorescent images in Figure 4 illustrates the spatial distribution of model API particles in the Nexthaler® aerosol during the first $25 \mathrm{~ms}$ after the start of the main aerosol plume emission. The test conditions were peak flow rate $Q_{\max }=60 \mathrm{I} \cdot \mathrm{min}^{-1}$ and rise time $T_{\text {rise }}=1.2 \mathrm{~s}$. Bright regions (coloured cyan) in the fluorescent image indicate high concentrations of microspheres. Figure 4 shows that the model API particles are concentrated near the walls of the mouthpiece exit, which matches the general structure of the powder aerosol described in Pasquali et al (2015). Very high brightness levels (coloured yellow/red), recorded during the period 0.23-0.238 s, are associated with a large and concentrated burst of model API fines coming out of the mouthpiece shortly after the actuation of the dose protector of the Nexthaler ${ }^{\circledR}$ DPI, which exposes the powder dose to the air stream when the suction pressure inside the device is $<-2 \mathrm{kPa}$. Subsequent images show lower and spatially more widely distributed brightness levels in the fluorescent image. Larger, very bright spots also start to appear in these images, which correspond to the emission of lower amounts of deaggregated model API fines; possibly carrier particles, covered in attached model API fines or larger self-agglomerates of model API.

\subsection{Effect of air suction profile: peak flow rate and rise time on model API fines emission}

The recorded image series for all suction conditions were analysed in order to show the temporal release of the model API fines by summing the pixel intensities in the analysis region defined in Figure 3 for each frame recorded; the results are shown in Figure 5 . The maximum fluorescent signal intensity was insensitive to the flow rate, but the timing of the peak intensity was found to vary with peak flow rate of the suction profile. The main fluorescent emission peak also appeared more quickly when the rise time was shorter. These trends are largely due to the dependence on suction profile of the timing of the $-2 \mathrm{kPa}$ trigger point for movement of the dose protector (Figure $4 \mathrm{~b}$ in Pasquali et al 2015). As a consequence, fluidisation of the powder dose will commence sooner after the start of the air flow when $\mathrm{Q}_{\max }$ is higher and/or $\mathrm{T}_{\text {rise }}$ is shorter. The start of the main emission peak was more erratic for 40 I.min ${ }^{-1}$ peak flows, because the steady-state pressure difference across the device is $2 \mathrm{kPa}$ at this flow rate. This is close to the trigger point for dose protector movement, so exposure of the powder dose to the air flow occurs haphazardly at this experimental condition. 


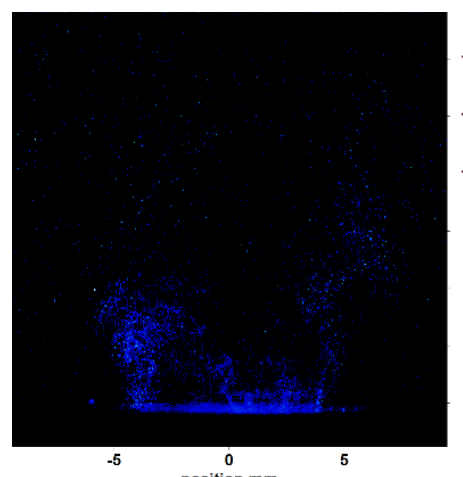

a) $0.124 \mathrm{~s}$

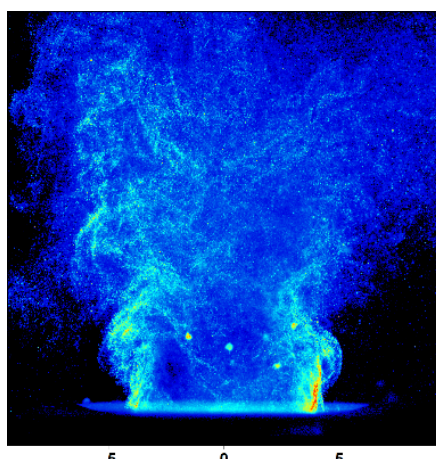

$\begin{array}{lc}-5 & 0 \\ & \text { position } \mathrm{mm}\end{array}$

d) $0.130 \mathrm{~s}$

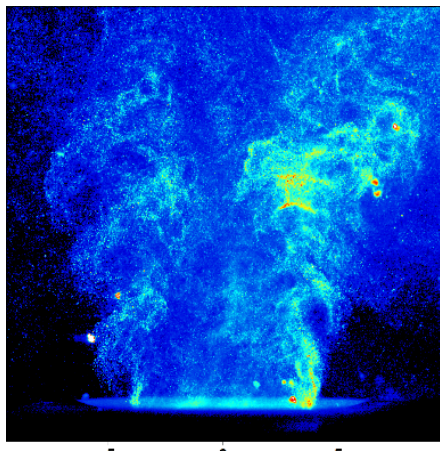

$-5 \quad$ position $\mathrm{mm}$

g) $0.136 \mathrm{~s}$

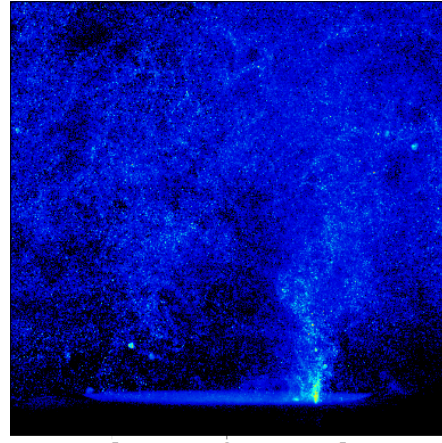

$-5$

j) $0.142 \mathrm{~s}$

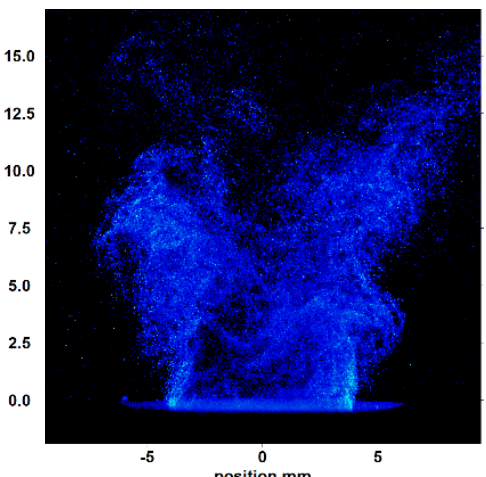

b) $0.126 \mathrm{~s}$

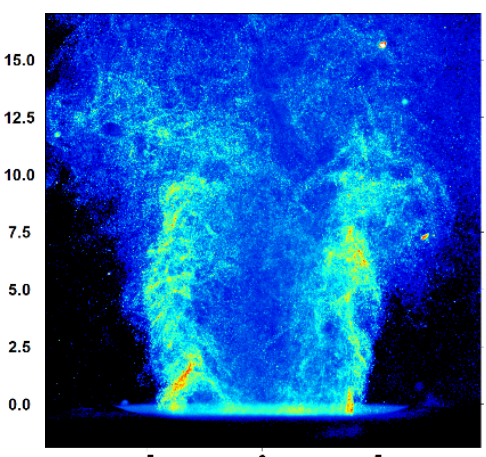

position $\mathrm{mm}$

e) $0.132 \mathrm{~s}$

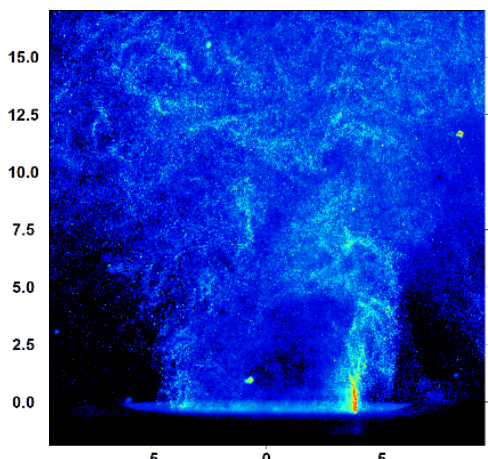

0
position $\mathrm{mm}$

h) $0.138 \mathrm{~s}$

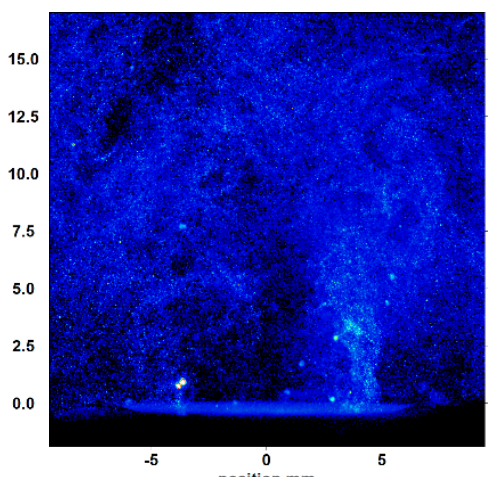

k) $0.144 \mathrm{~s}$

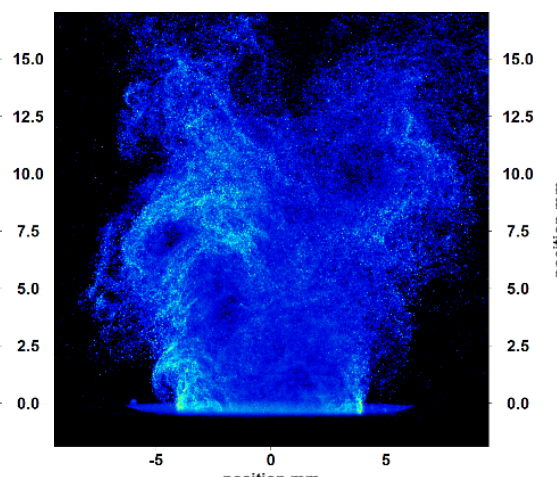

c) $0.128 \mathrm{~s}$

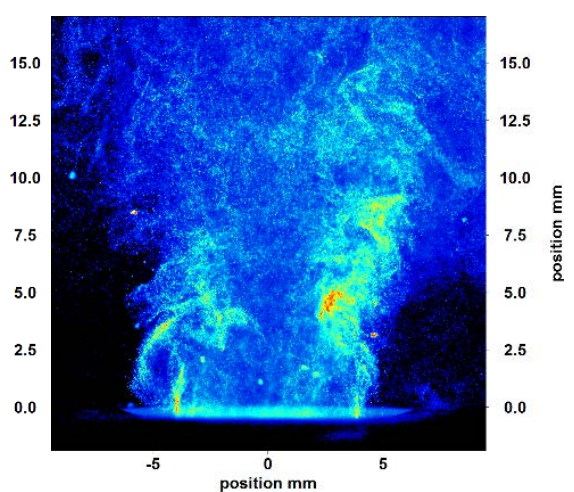

f) $0.134 \mathrm{~s}$

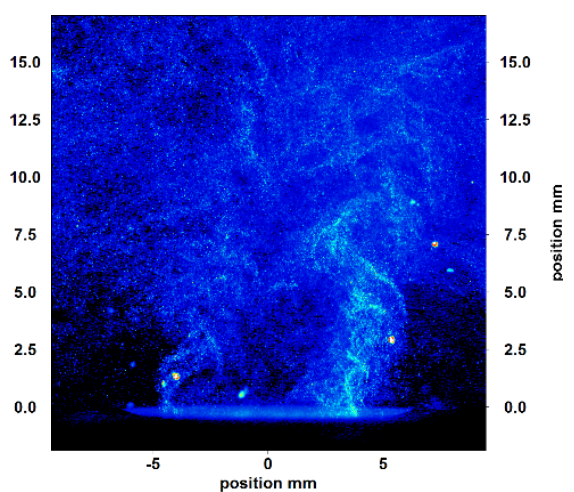

i) $0.140 \mathrm{~s}$

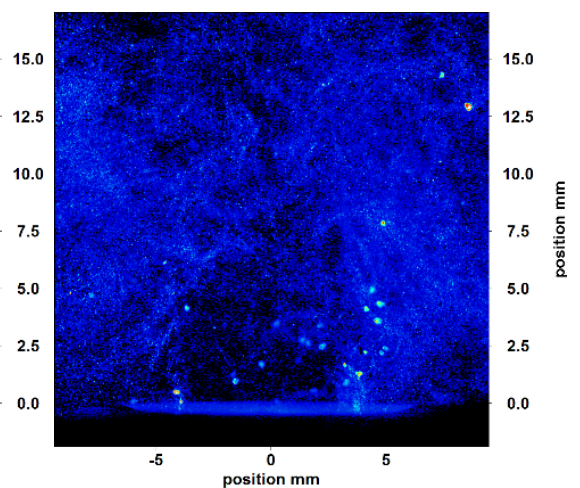

I) $0.146 \mathrm{~s}$

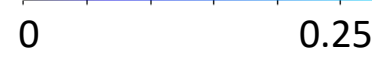

0.5

0.75

1

Light intensity, normalised to maximum

Figure 4: Fluorescent intensity mages in mouthpiece exit region of Nexthaler® during $25 \mathrm{~ms}$ after the start of powder emission (conditions: $1 \% \mathrm{w} / \mathrm{w}$ model API fines, $\mathrm{Q}_{\max }=60 \mathrm{~L} / \mathrm{min}, \mathrm{T}_{\text {rise }}=1.2 \mathrm{~s}$ ) 

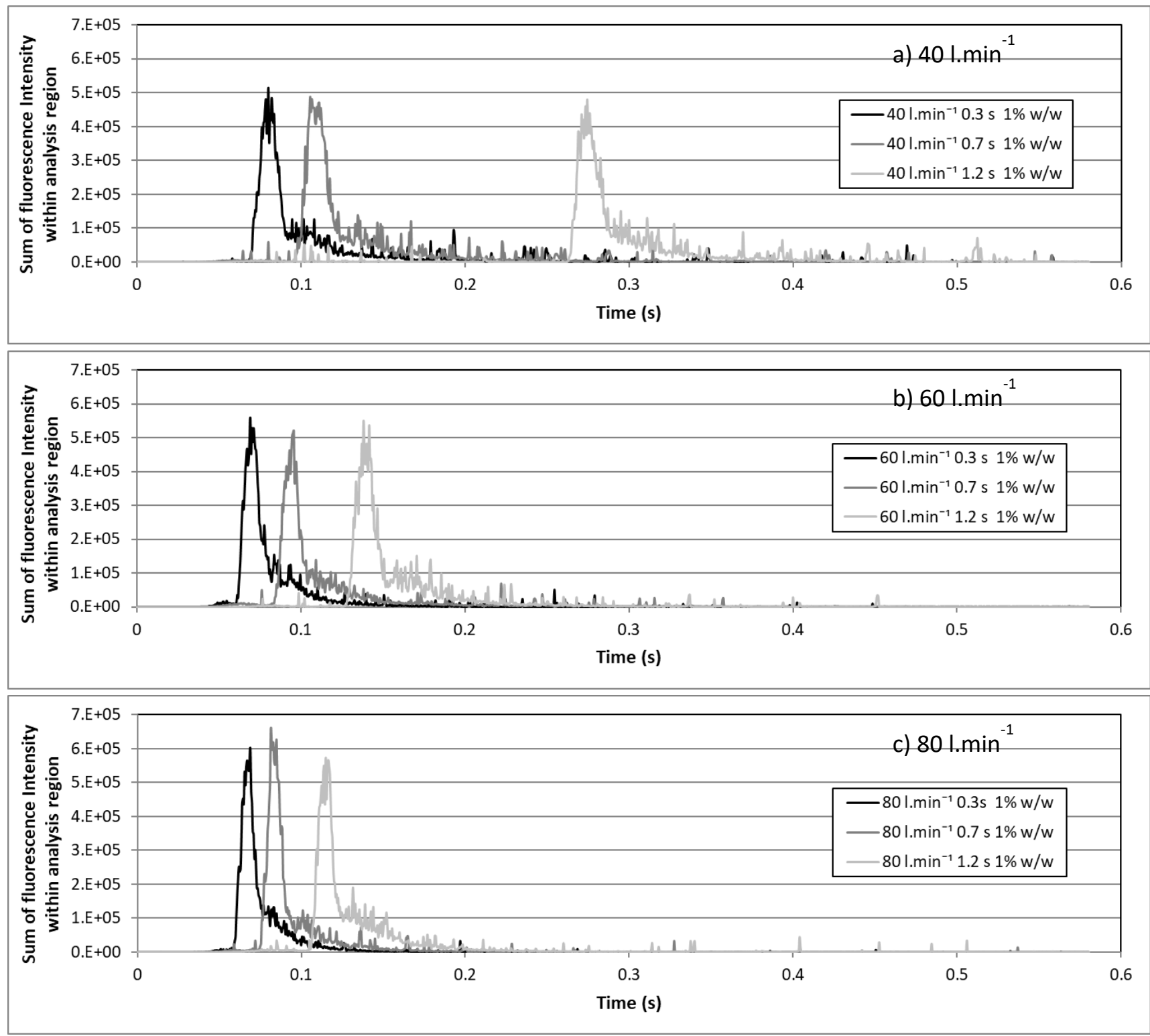

Figure 5: Fluorescent signal intensity count in analysis region as function of time for powder formulation with $1 \% \mathrm{w} / \mathrm{w}$ model API fines; air suction profiles with different suction rise time $\mathrm{T}_{\text {rise }}$ and peak flow rates (a) $\mathrm{Q}_{\max }=40 \mathrm{I} \cdot \mathrm{min}^{-1}$, (b) $\mathrm{Q}_{\max }=60 \mathrm{I} \cdot \mathrm{min}^{-1}$, (c) $\mathrm{Q}_{\max }=80 \mathrm{I} \cdot \mathrm{min}^{-1}$

\subsection{Scattered and fluorescent light images - emission of all powder vs. model API fines}

The degree of correlation between the emission of model API fines and the whole powder aerosol can be investigated by comparing pairs of simultaneous scattered light and fluorescent light images acquired by the two-camera optical setup. An example of such an image pair is given in Figure 6 . When examining image pairs, differences in the relative brightness patterns between fluorescent and scattered images should be interpreted with some care, since, even after the addition of a neutral density filter along the scattered light path to the camera, the dynamic range of the 
brightness levels of the fluorescent and scattered light images is not necessarily identical in the two images due to differences in the temporal release profiles of coarser powder fractions and model API fines.

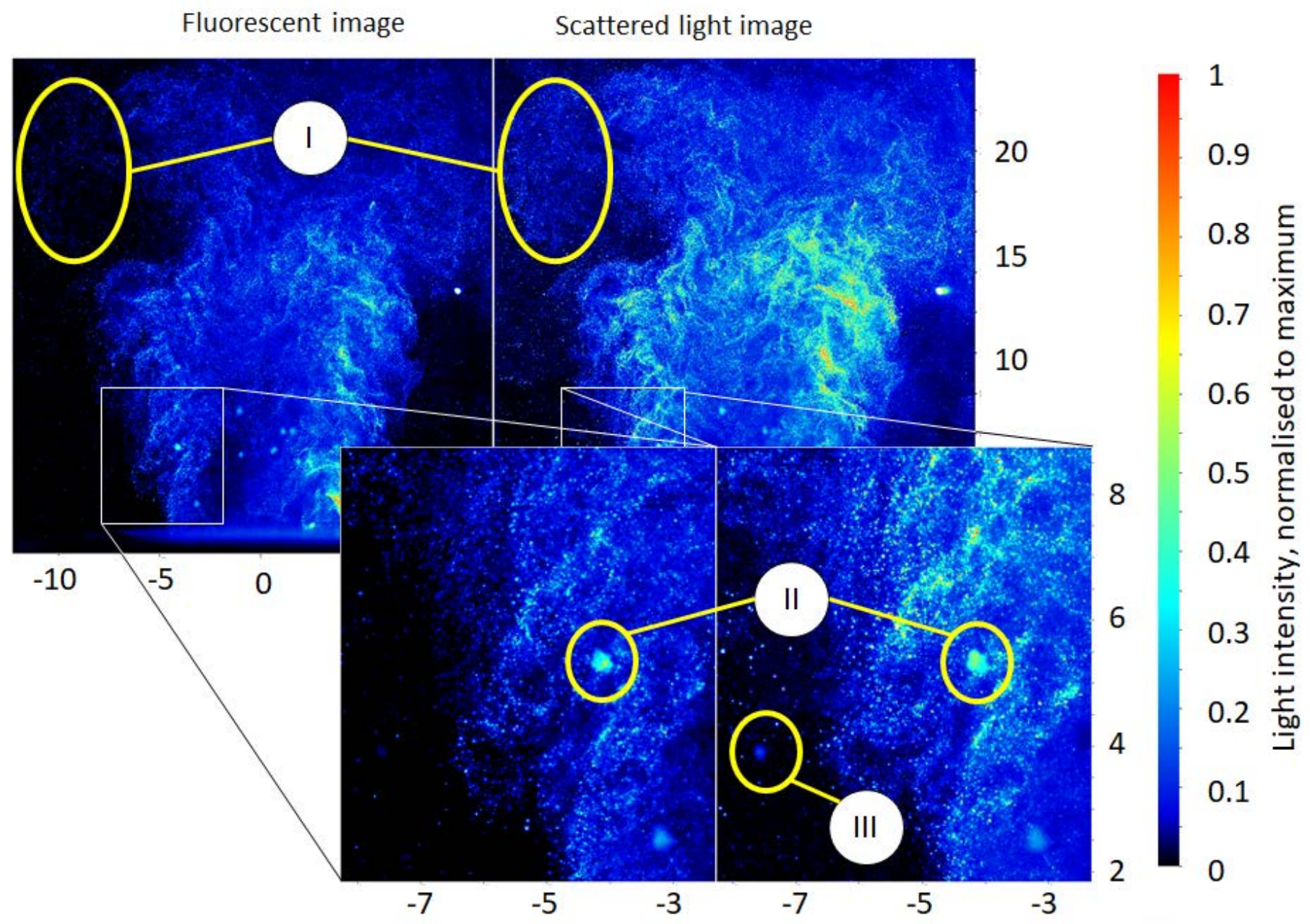

Figure 6 - Sample images from the simultaneous twin camera recording of aerosol plume just outside the mouthpiece exit; $(I)=$ region with low model API fines content; $(I I)=$ carrier particle with attached model API fines; (III) = carrier particle without model API fines

Figure 6 shows that the spatial distributions of brightness of the scattered and fluorescent light images of the aerosol plume are broadly similar. This suggests that the model API fines are well distributed throughout the powder, which is as expected, because high levels of turbulent mixing in the swirl chamber and device mouthpiece will mix fines thoroughly. There are, however, some bright regions in the scattered image, which do not appear to have a corresponding bright region in the fluorescent image (I) indicating a relatively low local concentration of model API fine. Concentrated bright spots in both fluorescent and scattered images (II) show the presence of large particles with 
significant fluorescent microspheres (model API) fines content, which could indicate lactose carrier particles with model API fines still attached to the surface or agglomerates of lactose and/or excipient fines with significant model API fines content. Large particles that appear fainter in the fluorescent image than in the scattered light image (III) represent carrier particles without attached microspheres or agglomerates of lactose/excipient fines without model API fines.

Figure 7 gives a sequence of image pairs acquired during an experiment with the following suction conditions: peak flow rate $60 \mathrm{I} \cdot \mathrm{min}^{-1}$ and rise time $1.2 \mathrm{~s}$. High brightness levels in concentrated regions were recorded in both image sets during a $10 \mathrm{~ms}$ time interval between $0.135 \mathrm{~s}$ and $0.145 \mathrm{~s}$ showing that release of most of the model API fines coincides with the main burst of powder emission, shortly after the actuation of the dose protector. After the rapid entrainment of the powder dose into the airflow, the powder is transported from the swirl chamber through a cylindrical outlet tube. The axial plane visualisation in Figure 7 and corresponding swirl-plane visualisation in Figure 8 both show that powder is most concentrated near the walls of the cylindrical tube due to the centrifugal forces acting on the particles, which travelled along spiralling paths as they emerge from the mouthpiece of the device (Pasquali et al, 2015).

After the main peak of powder emission, the fluorescent images show lower and more distributed brightness levels suggesting the emission of much lower amounts of de-aggregated model API fines during this phase of the powder fluidisation event. Small brighter spots in these images are associated with larger carrier particles and/or agglomerates of fines. These larger particles will experience a pronounced outward displacement and arrive at the mouthpiece later than the fines due to their higher inertia (see Figures 9 and 10).

The general similarity of the brightness patterns in the scattered and fluorescent images consistently appears in all other image sets acquired under different suction conditions. This confirms that model API fines are thoroughly mixed with the aerosol plume and that the emission of de-aggregated model API is largely correlated with emission of other fines (excipient and carrier). 


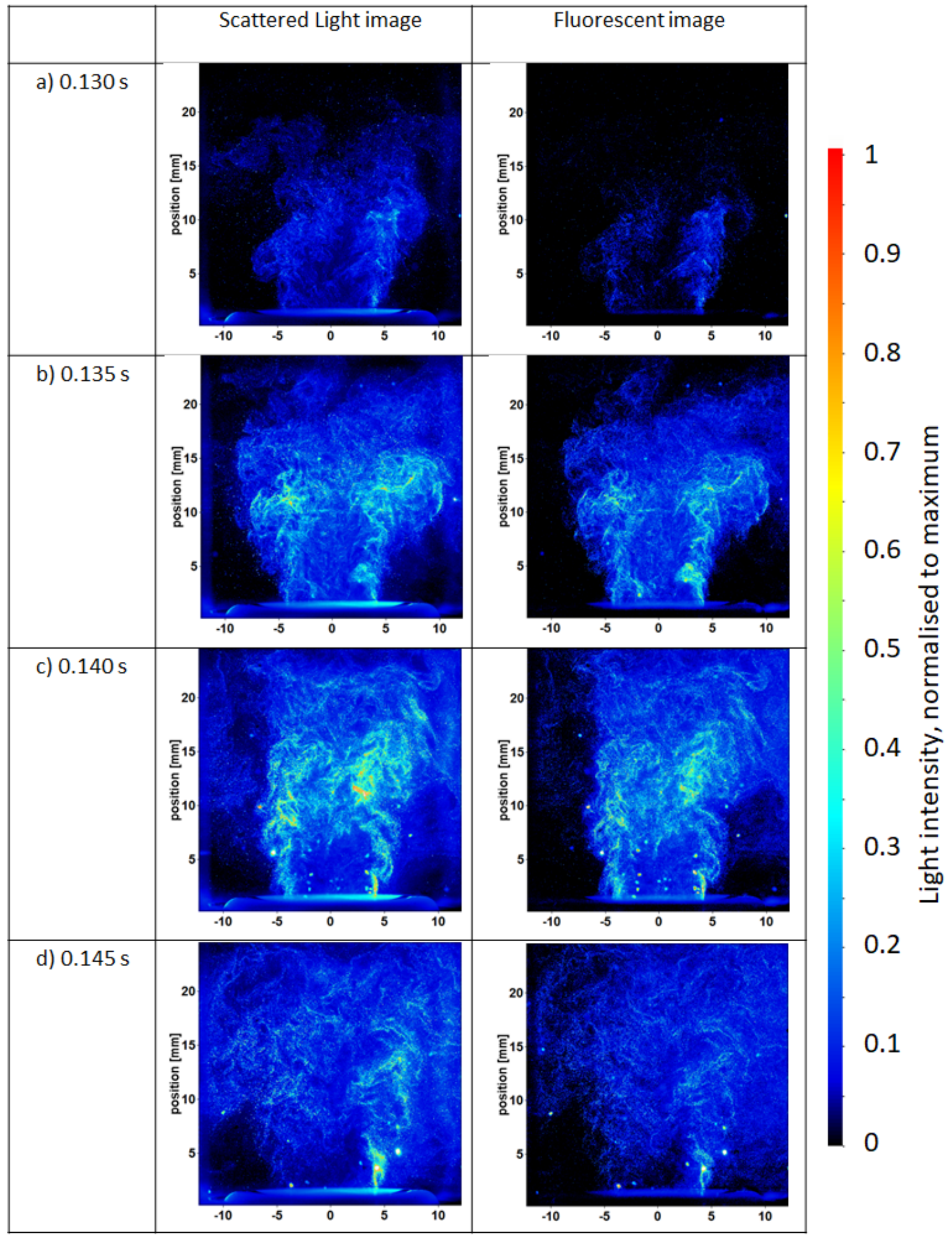

Figure 7: Axial plane visualisation of scattered and fluorescent intensity image pairs in mouthpiece exit region of Nexthaler ${ }^{\circledR}$ over a period of 15 ms representing the main fines emission (test conditions: $Q \max =60$ I $\cdot \min -1$, Trise $=1.2 \mathrm{~s}$ ) 
a) $0.1725 \mathrm{~s}$
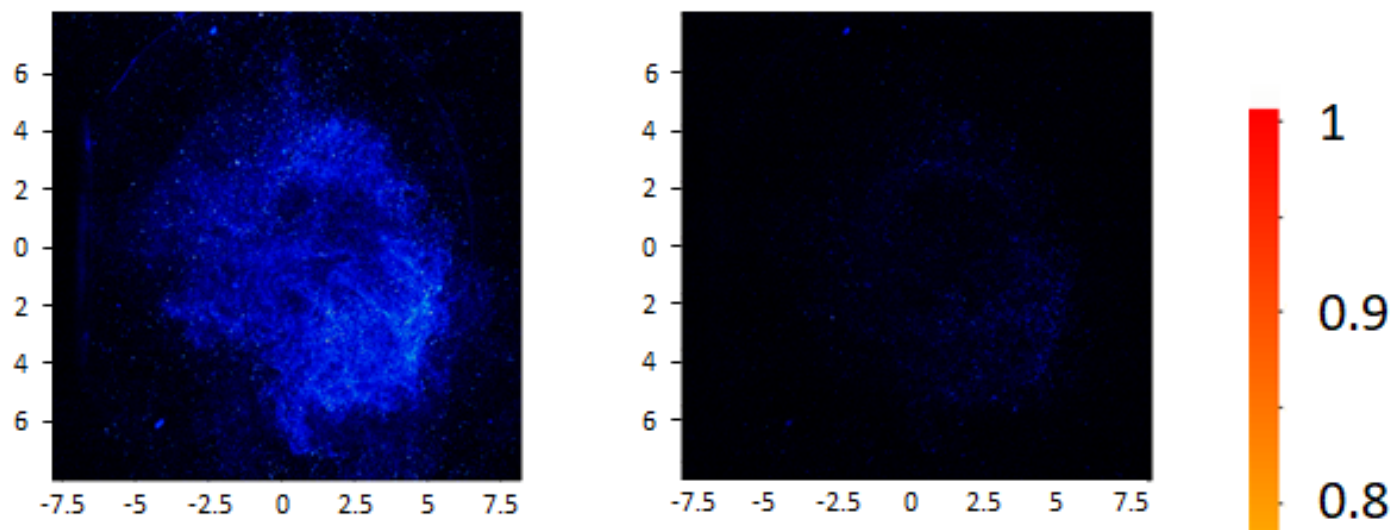

b) $0.1775 \mathrm{~s}$
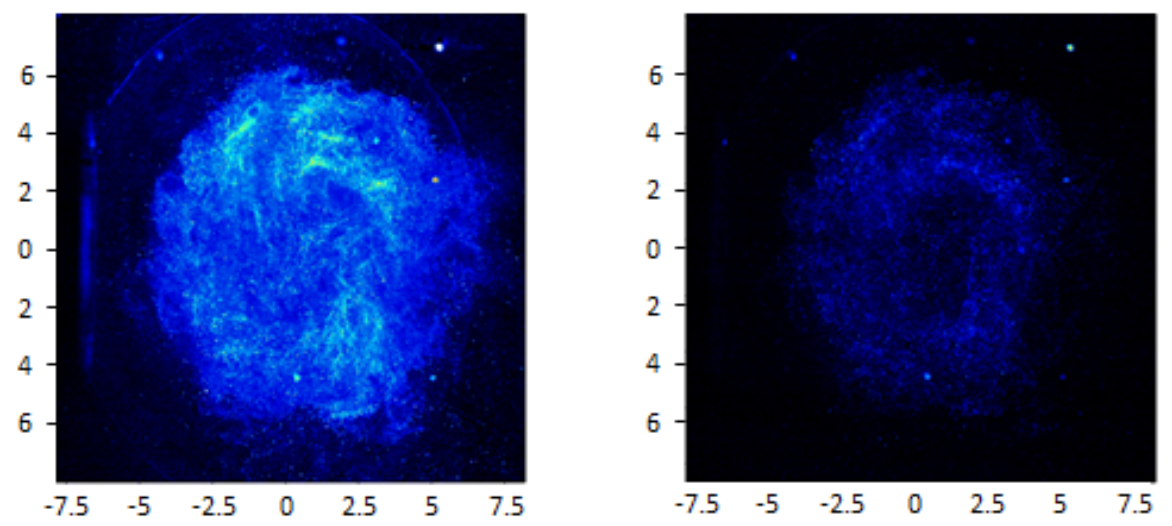

0.7

死

0.6

c) $0.1825 \mathrm{~s}$
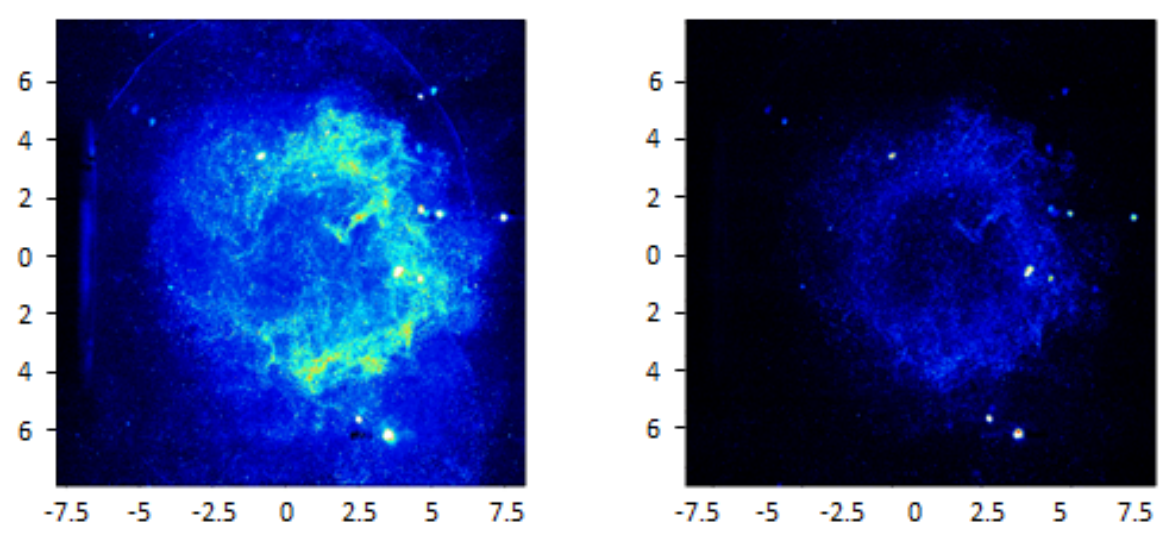

0.5



离

0.4

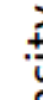

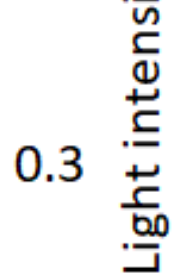

d) $0.1875 \mathrm{~s}$
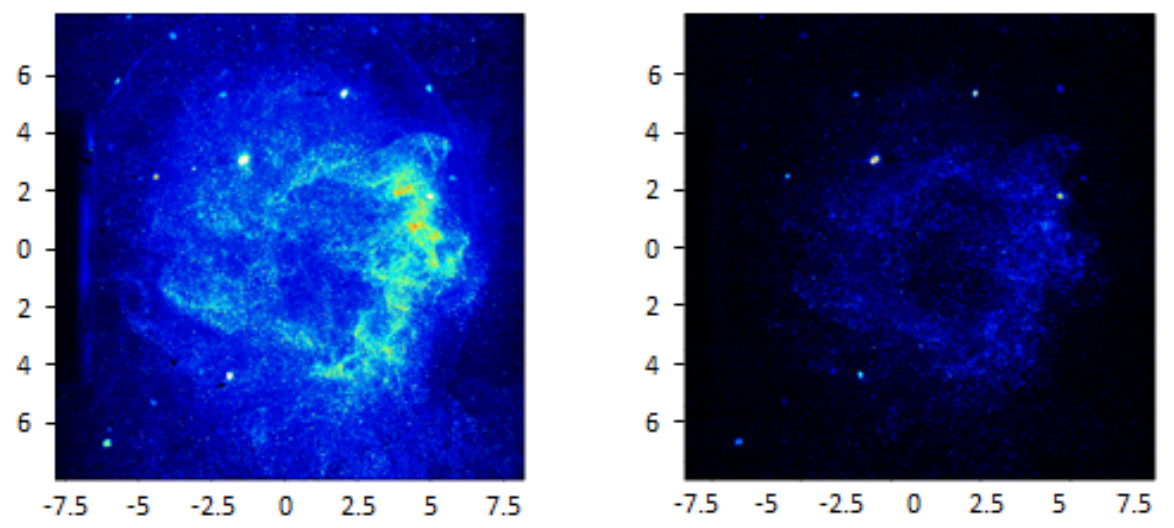

0.2

0.1

Figure 8: Swirl plane visualisation of scattered and fluorescent intensity image pairs in mouthpiece exit region of Nexthaler ${ }^{\circledR}$ over a period of 15 ms representing the main fines emission (test conditions: $Q \max =60$ I $\cdot \min -1$, Trise $=1.2 \mathrm{~s}$ ). 
The recorded fluorescent and scattered intensity image sets were analysed in order to compare the temporal release of the model API fines with the whole powder aerosol, respectively. The pixel intensities were summed in the analysis region defined in Figure 5 and normalised by the peak intensity as explained in section 2.2.4. Figure 9 compares the normalised fluorescent signal intensity (model-API only) with the normalised scattered signal intensity (all powder) for a series of experiments with a powder formulation containing $1 \% \mathrm{w} / \mathrm{w}$ concentration of model API in conjunction with a peak air flow rate of $60 \mathrm{I} \cdot \mathrm{min}^{-1}$ and rise times $0.3,0.7$ and $1.2 \mathrm{~s}$. The main features of the emission profiles were as follows:

A. Initial (premature) emission of particles early on in the suction profile as the airflow starts and before the 'breath activated mechanism' is triggered.

B. Main peak, just after the dose protector is withdrawn, releasing the formulation to the airflow.

C. Emission tail, reduced quantities of fine particles continue to leave the device and when most of the carrier particles leave the device.

D. Small intensity peaks after the main emission as occasional carrier particles and/or lactose/excipient agglomerates still leave the device.

In all of the traces, the shape of the main peak is nearly identical for the fluorescent and scattered intensity images. However, the emission tail is higher for the scattered light trace than the fluorescent light trace. The results of these experiments imply that the release of de-aggregated model API fines is concentrated toward the start of the powder fluidisation event and its release profile is identical to that of carrier particles and lactose/excipient fines, which represent the large majority of powder particles. Carrier particles and/or agglomerates of lactose/excipient fines are responsible for the larger emission peak in the scattered light signal during the emission tail. Most of the model API fines have been released earlier, so the emission tail in the fluorescent signal is

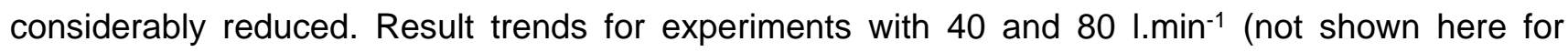
brevity) were found to be very similar. 

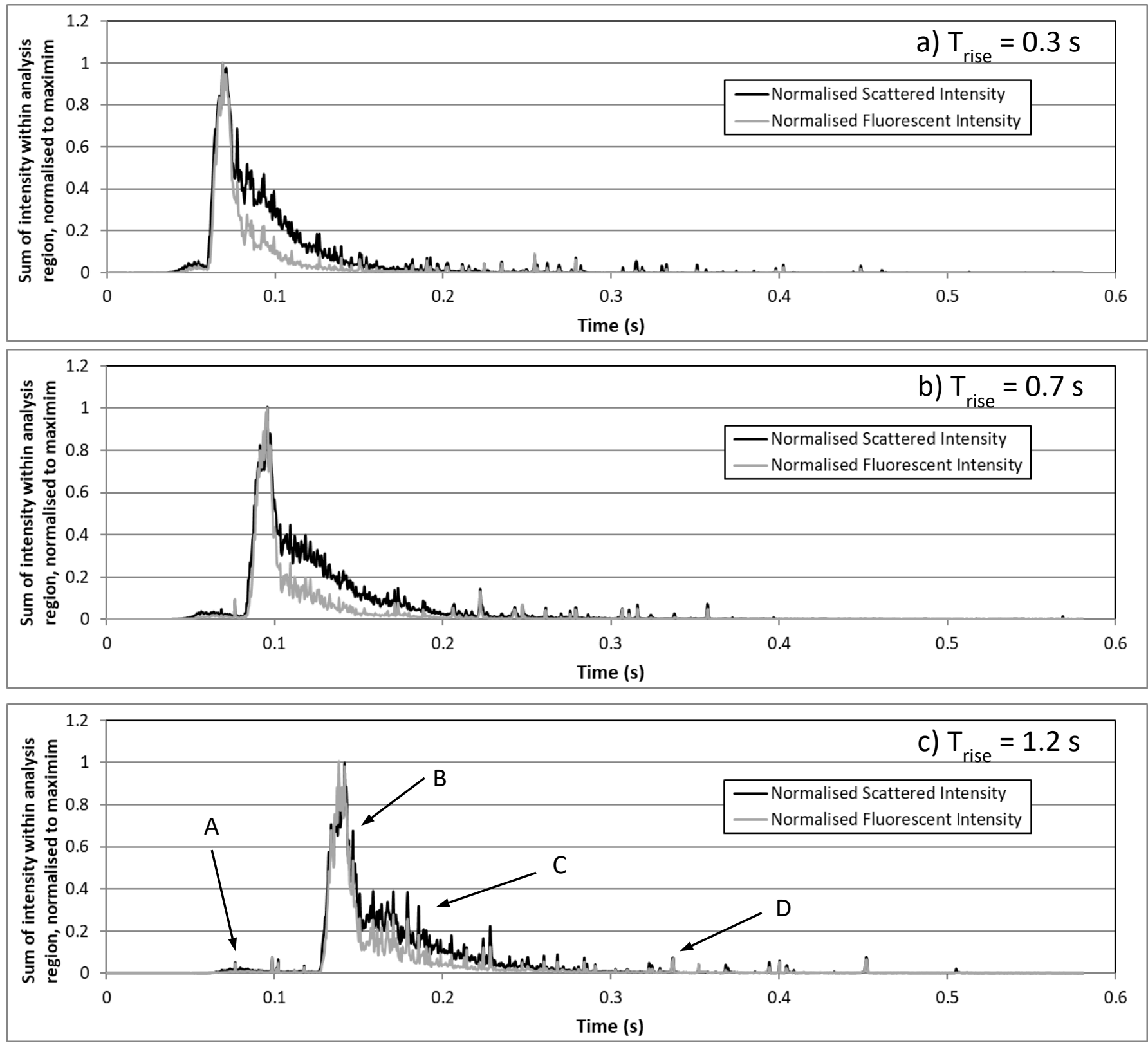

Figure 9: Comparison of fluorescent signal intensity and scattered signal intensity in analysis region for suction profiles with $\mathrm{Q}_{\max }=60 \mathrm{I} \cdot \mathrm{min}^{-1}$ (a) $\mathrm{T}_{\text {rise }}=0.3 \mathrm{~s}$, (b) $\mathrm{T}_{\text {rise }}=0.7 \mathrm{~s}$, (c) $\mathrm{T}_{\text {rise }}=1.2 \mathrm{~s}$

The overall duration of the aerosol plume emission was found by determining the time from the start of the main intensity peak to the moment when $90 \%$ of the aerosol plume was emitted by numerical integration of the intensity-time profiles. The results of these calculations for all flow conditions are given in Table 2. The release of the fluorescent particles (model API) is completed earlier than the whole powder dose. The difference between the release times of model API and the whole powder was found to be greater for higher peak flow rates and shorter rise times. More flow energy is available for de-aggregation of fines from carrier particles when the flow changes are more abrupt, causing the model API to separate from the carrier particles earlier in the release process. 


\begin{tabular}{|c|c|c|c|c|}
\hline $\begin{array}{l}\text { Steady } \\
\text { state air } \\
\text { flow rate } \\
\left(1 . \mathrm{min}^{-1}\right)\end{array}$ & $\begin{array}{l}\text { Rise time of } \\
\text { ramp profile } \\
\text { (s) }\end{array}$ & $\begin{array}{c}\text { Time } T_{\text {API }} \text { to release } \\
90 \% \text { of fluorescent } \\
\text { (model API) content } \\
\text { (ms) }\end{array}$ & $\begin{array}{c}\text { Time } \mathrm{T}_{\text {all }} \text { to release } \\
90 \% \text { of whole } \\
\text { formulation } \\
(\mathrm{ms})\end{array}$ & $\begin{array}{c}\text { Difference } \mathrm{T}_{\text {all }}-\mathrm{T}_{\mathrm{API}} \\
(\mathrm{ms})\end{array}$ \\
\hline 40 & 0.3 & 136.5 & 151.0 & 14.5 \\
\hline 40 & 0.7 & 132.0 & 143.5 & 11.5 \\
\hline 40 & 1.2 & 126.5 & 129.5 & 3.0 \\
\hline 60 & 0.3 & 58.5 & 78.0 & 19.5 \\
\hline 60 & 0.7 & 87.5 & 101.5 & 14.0 \\
\hline 60 & 1.2 & 94.0 & 102.5 & 8.5 \\
\hline 80 & 0.3 & 40.5 & 62.0 & 21.5 \\
\hline 80 & 0.7 & 56.5 & 71.0 & 14.5 \\
\hline 80 & 1.2 & 74.0 & 82.5 & 8.5 \\
\hline
\end{tabular}

Table 2: Time to release of $90 \%$ of model API content and $90 \%$ of whole powder dose (powder formulation: $1 \% \mathrm{w} / \mathrm{w}$ model API fines)

\subsection{Effect of concentration of fluorescent particles}

A series of experiments was carried out to study the effect of variations of the model API content spanning the range of practical API concentrations found in typical DPI formulations. The main purpose was to understand whether the temporal profile of API release was affected by fluorescent particle concentration. Chiesi's proprietary Nexthaler ${ }^{\circledR}$ carrier and excipient were blended with fluorescent microspheres to produce the following nominal model-API w/w concentrations: $0.02 \%$, $0.05 \%, 0.25 \%, 1 \%$ and $2 \%$. After loading into Nexthaler ${ }^{\circledR}$ prototype DPIs, these formulations were tested with air suction conditions corresponding to peak flow rates of 40,60 and 80 I.min $^{-1}$ in conjunction with the intermediate rise time of $0.7 \mathrm{~s}$. Separate inhaler devices were used for the testing of each formulation, resulting in small variations in the triggering of the dose release.

The temporal development of each of the aerosol plumes generated during these tests was very similar to those described in the previous section. Figure 10 shows typical fluorescent signal intensity traces in the analysis region as a function of time for the experiments conducted with a 
peak flow rate of $80 \mathrm{I} \cdot \mathrm{min}^{-1}$. The maximum signal intensity was found to be insensitive to the peak flow rate, but the timing of the maximum intensity again varied somewhat with air flow rate and rise time, which was in general agreement with the results for powder emission of placebo formulation (without active or model API) described in Pasquali et al (2015).

The peak fluorescent intensity increases with fluorescent particle concentration as expected (maximum of $\sim 10^{4}$ counts for $0.02 \%$ w/w concentration in Fig. 10 .a up to $1.6 \times 10^{6}$ for the $2 \%$ w/w concentration in Fig. 10.e). The results of quantitative analysis of the fluorescent signal intensity obtained from analysis of the rectangular $16 \mathrm{~mm} \times 4 \mathrm{~mm}$ analysis region just outside the mouthpiece exit are given in Table 3.

\begin{tabular}{|c|c|c|c|}
\hline $\begin{array}{c}\text { Nominal concentration of } \\
\text { fluorescent particles (w/w) }\end{array}$ & \multicolumn{3}{|c|}{ within the analysis region } \\
\hline 0.02 & 151 & 129 & 104 \\
\hline 0.05 & 302 & 201 & 193 \\
\hline 0.25 & 2000 & 1590 & 1684 \\
\hline 1.00 & 12290 & 10521 & 13779 \\
\hline 2.00 & 27356 & 40419 & 28842 \\
\hline Flow rate $\left(\mathrm{l} . \mathrm{min}^{-1}\right)$ & 40 & 60 & 80 \\
\hline
\end{tabular}

Table 3: Total fluorescent intensity count for different peak flow rates and fluorescent microsphere concentrations after aerosolisation of lactose blend 

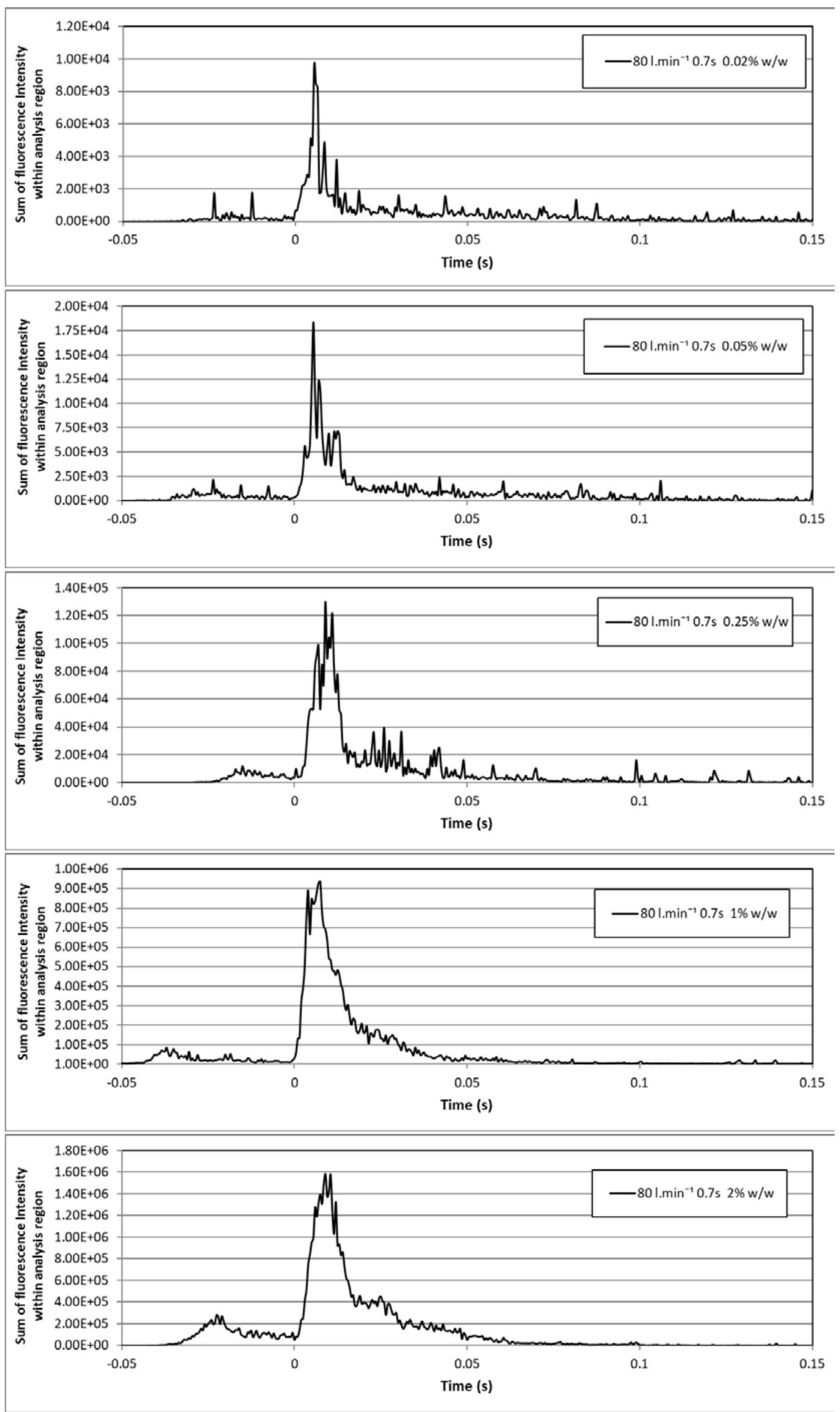

Figure 10.a-e - Fluorescent light intensity traces for model-API concentrations between $0.02 \% \mathrm{w} / \mathrm{w}$ and $2 \% \mathrm{w} / \mathrm{w}$ at peak flow rate of $80 \mathrm{I} \cdot \mathrm{min}^{-1}$ and rise time of $0.7 \mathrm{~s}$. 
The area under the fluorescent intensity traces represents the total pixel intensity count for each test condition. Table 3 shows that, for a fixed fluorescent particle concentration, the total intensity count was found to vary somewhat with peak flow rate. Furthermore, the data in Table 3 highlight that the total intensity count increases as the concentration of fluorescent microspheres goes up. The total intensity is not exactly proportional to concentration and independent of flow rate due to variations that occur between experiments, most notably, variation in laser power affects the light intensities received by the cameras during different experiments. This effect can be reduced by considering the ratio of the total fluorescent intensity count and the total scattered intensity count during the same fluidisation experiment. This normalisation is useful, because variations of the laser light intensity between different tests affect the pixel intensities for whole powder dose and the fines in the same way.

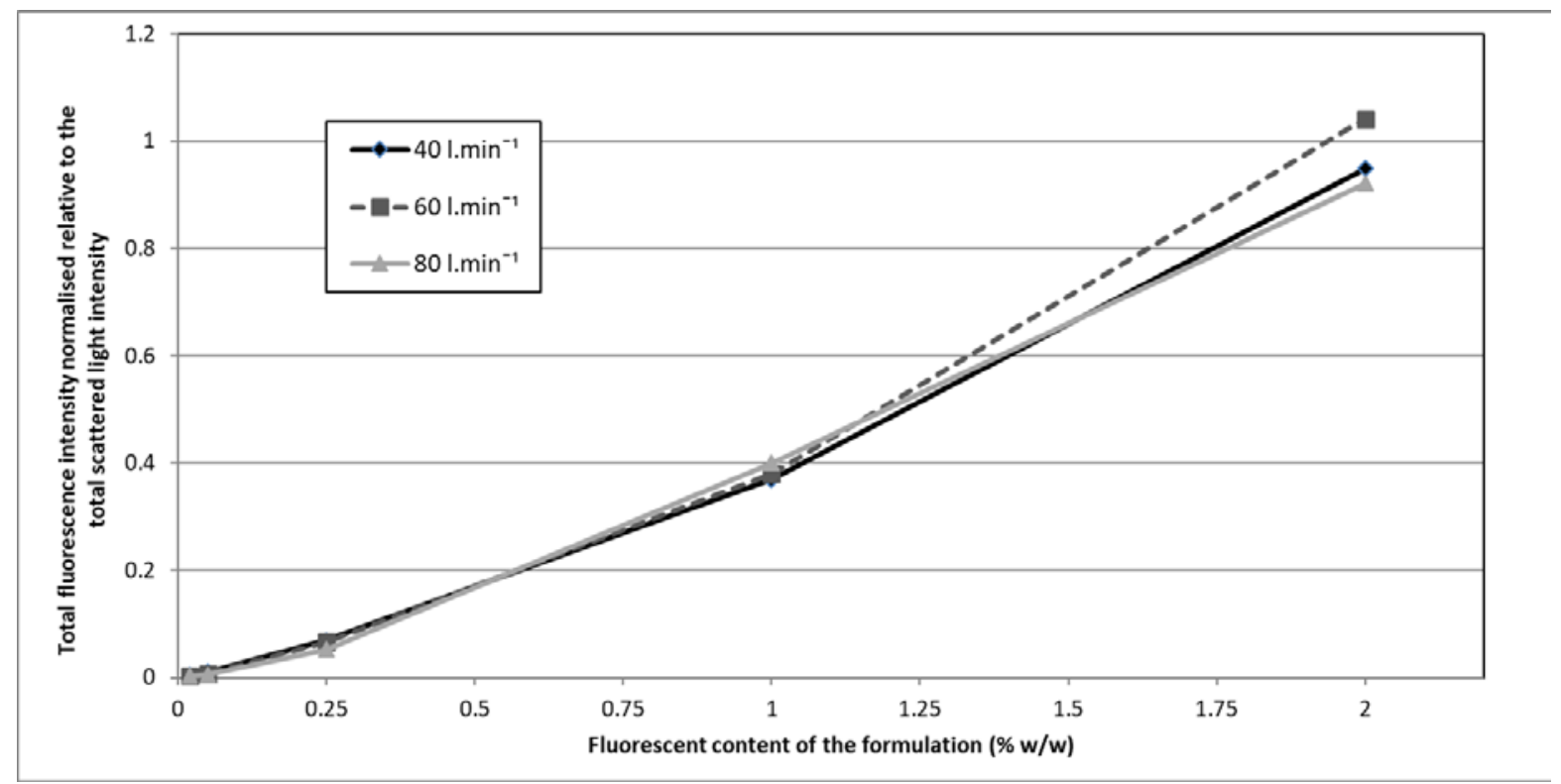

Figure 11: Fluorescent signal intensity normalised by scattered light intensity vs. \% w/w concentration of fluorescent microspheres

Figure 11 shows that the relationship between the normalised fluorescent light intensity and the \% w/w concentration of fluorescent microspheres is almost linear. If the fluorescent signal intensity was dependent purely on the number of fluorescent particles in the formulation, the expected relationship would be a straight-line intersecting the origin. For dispersed aerosols with sufficiently large interparticle distance, the contribution due to de-aggregated fines should be proportional to the number concentration of model API fines, hence, linearly proportional to the $\% \mathrm{w} / \mathrm{w}$ of model-API. The most likely cause of departures from linear behaviour is loss of visible particles. This may be caused by: 
- $\quad$ Fines deposition inside the device passages.

- Incomplete de-aggregation causing a fraction of the microspheres to remain attached to carrier particles.

Even a small number of fluorescent microspheres lost in this way could cause a substantial drop in recorded fluorescence signal at lower concentrations. An indication that fines attached to larger carrier particles may be responsible for this effect, is found in the large and rapid intensity fluctuations towards the end of the main peak in the intensity-time traces corresponding to lower model-API concentrations (see Figs 10.a-b). Traces corresponding to high model-API concentration results are much more smooth (see Fig 10.e). De-aggregated fines are rapidly dispersed throughout the aerosol and will generate smoothly varying fluorescent intensity-time traces. Pasquali et al (2015) suggested that spikes in the later part of the scattered light intensity-time traces are associated with the emission of individual carrier particles. At the lowest concentration of model API fines, a larger proportion of model API fine particles remains attached to such large particles, which would explain the large fluorescent intensity spikes.

The observed trends are in agreement with the theory of blend structure and influence of drug loading on fine-particle dose in carrier formulations proposed by Young et al (2011). During blending, the sites on carrier particles with the strongest adhesion (i.e. highest adhesion energy) are populated first by fines. As the concentration of drug fines increases, sites with lower adhesion energy will be populated next. At very high fines concentrations, all sites will be filled with drug fines and additional fines will adhere to already-attached drug fines, forming a second attached layer. After fluidisation, the aerosol plume will mainly contain the most loosely-bound fines originating from sites with low adhesion energy. The fine particles on the sites with high adhesion energy are more likely to remain attached. Assuming that the number of such high adhesion sites is uniformly distributed amongst carrier particles, this loss of strongly-adhered fines will cause a disproportionately large effect on the fluorescent intensity traces when the concentration of fluorescent microspheres is low. Since only one side of each carrier particle is visible to the camera, a substantial fraction of the attached fines remains invisible to the camera. This could explain the disproportionate reduction of the normalised fluorescent signal intensity to reduce at low concentrations and, hence, the non-linear relationship between fluorescent intensity and model API concentration. 


\section{Concluding Remarks}

The fluorescent imaging system described in this paper has been shown to provide an effective method for characterising the release of model API fines from a dry powder inhaler under different flow rate profiles and different model API concentrations. The Nexthaler ${ }^{\circledR}$ DPI is designed to deliver consistent powder aerosols with extrafine (1-2 $\mu \mathrm{m})$ particle content with minimal effect of inhaled air flow rate. A key design feature is its dose protector, which prevents powder emission until the suction pressure of the inhaled air exceeds $-2 \mathrm{kPa}$. This ensures that the air flow has accelerated sufficiently and reduces the dependence of delivered drug dose on a patient's inspiratory effort. Buttini et al (2015) and Buttini et al (2016) considered this issue and produced a significant dataset to confirm that the performance of the Nexthaler ${ }^{\circledR}$ device was insensitive to applied air flow rate. The fluorescent imaging system has provided further confirmation of these findings, showing that the API fines are emitted efficiently for a range of different suction conditions and model API concentrations that are representative of practical formulations.

Furthermore, the fluorescent imaging technique has given detailed information relating to the temporal profile and the spatial distribution of model API fines during their emission. The release of a large fraction of the model API occurred in a short $10-20$ ms burst after the start of the particle emission event; the remainder was released more gradually over a 100-200 ms period. The pattern of release of the model API was substantially independent of suction conditions, but, for suction profiles with more rapid flow acceleration (shorter rise time and higher peak flow) the $-2 \mathrm{kPa}$ suction pressure needed to trigger movement of the dose protector of the Nexthaler® DPI is reached earlier. Thus, the powder dose is fluidised more quickly after the start of suction, leading to faster release of the API fines.

The fluorescent imaging technique gave consistent results across a wide range of model API content without the need to change filters, lens settings, or laser power. The two-camera arrangement enables direct comparison of the release of the model API and the whole formulation. Test results have shown that, while most of the model-API particles ares released from the carrier particles during the entrainment process, in all cases some of large particles with significant fluorescent microsphere content were observed. The total fluorescence intensity, normalised by the total scattered light intensity, increases with fluorescent particle concentration in an almost linear 
fashion. Reduced intensity occurs for low concentration formulations. The reduced visibility of the model API fines at low concentrations follows trends that are also predicted by a model of the effect of drug loading on FPF proposed by Young et al (2011).

The work reported in the present manuscript demonstrates the merits of fluorescent imaging using prototypes of the Nexthaler® DPI and proprietary development placebo formulation. However, the optical diagnostics and digital image analysis techniques are completely generic and can be used for detailed fundamental study of powder fluidisation inside a DPI device by making use of transparent model devices. Moreover, the methods can also be applied during development of DPI products as soon as realistic prototype devices are available. Future studies should realise the potential of this optical diagnostic technique by further in-depth image analysis to reveal details of the processes leading to drug fines emission in model formulations containing coarse lactose carrier and fluorescent microspheres.

\section{References}

- Buttini, F., Pasquali, I., Brambilla, G., Copelli, D., Alberi, M. D., Balducci, A. G., Bettini, R., Sisti, V. (2015). 'Multivariate analysis of effects of asthmatic patient respiratory profiles on the in vitro performance of a reservoir multidose and a capsule-based dry powder inhaler', Pharmaceutical Research, Vol. 33, pp. 701-715.

- Buttini F., Brambilla G., Copelli D., Sisti V., Balducci A.G., Bettini R., Pasquali I. (2016). 'Effect of flow rate on in vitro aerodynamic performance of NEXThaler® in comparison with Diskus ${ }^{\circledR}$ and Turbohaler ${ }^{\circledR}$ dry powder inhalers', Journal of Aerosol Medicine and Pulmonary Drug Delivery, Vol. 29, No. 2, pp. 167-178.

- Chan H.-K. and Chew N.Y.K. (2003). 'Novel alternative methods for the delivery of drugs for the treatment of asthma', Advanced Drug Delivery Reviews, Vol. 55, pp. 793-805.

- Coates, M.S., Chan, HK., Fletcher, D.F., Raper, J.A. (2005), 'Influence of air flow on the performance of a dry powder inhaler using computational and experimental analyses', Pharmaceutical Research, Vol. 22, No. 9, 1445-1453. 
- Corradi M., Chrystyn H., Cosio B.G., Pirozynski M., Loukides S., Louis R., Spinola M., Usmani O.S. (2014). 'Nexthaler, an innovative dry powder inhaler delivering an extrafine combination of beclomethasone and formoterol to treat large and small airways in asthma', Expert Opinion on Drug Delivery, Vol. 11, Issue 9, pp. 1497-1506.

- Cospheric Inc. (2018). 'FMR red fluorescent microspheres manufacturer's data', https://www.cospheric.com/FMR red fluorescent polymer microspheres 1micron.htm , Accessed 20-06-2018.

- European Directorate for Quality in Medicines and Healthcare (2013): Preparations for inhalation: Aerodynamic assessment of fine particles. General Chapter 2.9.18, European Pharmacopoeia, $7^{\text {th }}$ ed, pp. 278-282.

- Fergenson D.P., Morrical B.D., Balaxi M., and Brand N. (2013). 'Real-Time Determination of Aerodynamic Particle Size Profiles and the Presence/Co-Presence of Active Ingredients in Inhalational Pharmaceutical Products Using a SPAMS 3.0', Proceedings of RDD Europe 2013, Vol. 2, pp. 317-320.

- Finlay W.H. (2001). 'The mechanics of inhaled pharmaceutical aerosols: an introduction', Academic Press, San Diego.

- Jetzer M.W., Morrical B.D., Schneider M., Edge S. and Imanidis G. (2017). 'Probing the particulate microstructure of the aerodynamic particle size distribution of dry powder inhaler combination products', International Journal of Pharmaceutics (2010), https://doi.org/10.1016/j.ijpharm.2017.12.046.

- Laube B.L., Janssens H.M., de Jongh F.H.C., Devadason S.G., Dhand R., Diot P., Everard M.L., Horvath I., Navalesi P., Vosbaar T. and Chrystyn H. (2011). 'ERS/ISAM task force consensus statement: recommendations on what pulmonary specialist should know about the new inhalation therapies, European Respiratory Journal, Vol. 37, pp. 1308-1331.

- Merzkirch W. (1987). 'Flow visualization', $2^{\text {nd }}$ ed, Academic Press, Orlando, FL, USA.

- Morrical B.D., Balaxi M., Fergenson D. (2015). 'The on-line analysis of aerosol-delivered pharmaceuticals via single particle aerosol mass spectrometry', International Journal of Pharmaceutics 489, pp. 11-17.

- Newman S.P. and Busse W.W. (2002). 'Evolution of dry powder inhaler design, formulation and performance', Respiratory Medicine, Vol. 96, 293-304. 
- $\quad$ Ngoc N.T.Q., Chang L., Jia X., Lau R. (2013). ‘Experimental investigation of design parameters on dry powder inhaler performance', International Journal of Pharmaceutics, Vol. 457, pp. 92-100.

- Nichols S.C. and Wynn E. (2008), 'New approaches to optimizing dispersion in dry powder inhalers — dispersion force mapping and adhesion measurements', Respiratory Drug Delivery 2008, Vol. 1, pp. 175-184.

- Pasquali I., Brambilla G., Long E.J., Hargrave G.K. and Versteeg H.K. (2012). 'A visualisation study for the aerosol generation in NEXThaler®', Poster abstract MN-1150, Proceedings AAPS Conference, October 2012, Chicago, IL.

- Pasquali I., Merusi C., Brambilla G., Long E.J., Hargrave G.K. and Versteeg H.K. (2015). 'Optical diagnostics study of air flow and powder fluidisation in Nexthaler1—Part I: Studies with lactose placebo formulation', International Journal of Pharmaceutics 496, pp. 780-791.

- Sim S., Margo K., Parks J., Howell R., Hebbink G.A., Orlando L., Leslie P., Ho L., Morton D.A.V. (2014). 'An insight into powder entrainment and drug delivery mechanisms from a modified Rotahaler', International Journal of Pharmaceutics, Vol. 477, pp. 351-360.

- Susz A., Morrical B.D., Fergenson D.P. (2014). 'New determination method of aerodynamic diameter size distribution in dry powder inhalers using SPAMS 3.0', In: Dalby RN, Byron PR, Peart J, Suman JD, Traini D. and Young PM, (eds). Respiratory Drug Delivery 2014, Davis Healthcare International Publishing LLC, River Grove, Illinois, USA; pp. 515-518, 2014.

- $\quad$ Towers D.P., Towers C.E., Buckberry C.H., Reeves M. (1999). 'A colour PIV system employing fluorescent particles for two-phase flow measurements', Measurement Science and Technology, Vol. 10, pp. 824-830.

- Tuley R., Shrimpton J., Jones M.D., Price R., Palmer M., Prime D. (2008). ‘Experimental observation of dry powder inhaler dose fluidisation', International Journal of Pharmaceutics, Vol. 358, pp. 238-247.

- United States Pharmacopoeia: USP37-NF 30, Chapter 601: Physical tests and determinations; Aerosols, 2013. ISBM-9781936424221.

- Voss, A., Finlay, W.H. (2002). 'Deagglomeration of dry powder pharmaceutical aerosols'. International Journal of Pharmaceutics, Vol. 248, pp. 39-50. 
- Young P.M., Wood O., Ooi J., Traini D. (2011)."The influence of drug loading on formulation structure and aerosol performance in carrier based dry powder inhalers", International Journal of Pharmaceutics, Vol. 416, pp. (2011) 129- 135.

- Zeng X.-M., MacRitchie H.B., Marriott C., Martin G.P. (2006). 'Correlation between inertial impaction and laser diffraction sizing data for aerosolized carrier-based dry powder formulations', Pharmaceutical Research, Vol. 23, No. 9, pp. 2200-2209.

- Zhou Q., Tang P., Leung S.S.Y., Chan J.G.Y., Chan H.-K. (2014), 'Emerging inhalation aerosol devices and strategies: where are we headed?', Advanced Drug Delivery Reviews, Vol. 75, pp. 3-17. 